\title{
Thermal deoxygenation causes Photoluminescence shift from UV to blue region in lyophilized graphene oxide
}

\author{
Veeresh kumar $^{1,2 *}$, Vivek Kumar ${ }^{1,3}$, G. B. Reddy ${ }^{1}$ and Renu Pasricha ${ }^{2{ }^{*}}$ \\ ${ }^{1}$ Department of Physics, Indian Institute of Technology New Delhi-110016, India \\ ${ }^{2}$ CSIR-National Physical Laboratory new Delhi-110012, India \\ ${ }^{\dagger}$ Present Address: National Centre for Biological Sciences-TIFR, Bangalore \\ ${ }^{3}$ Department of Physics, National Institute of Technology Meghalaya, Laitumkhrah Shillong - \\ 793003, Meghalaya, India (Present address)
}

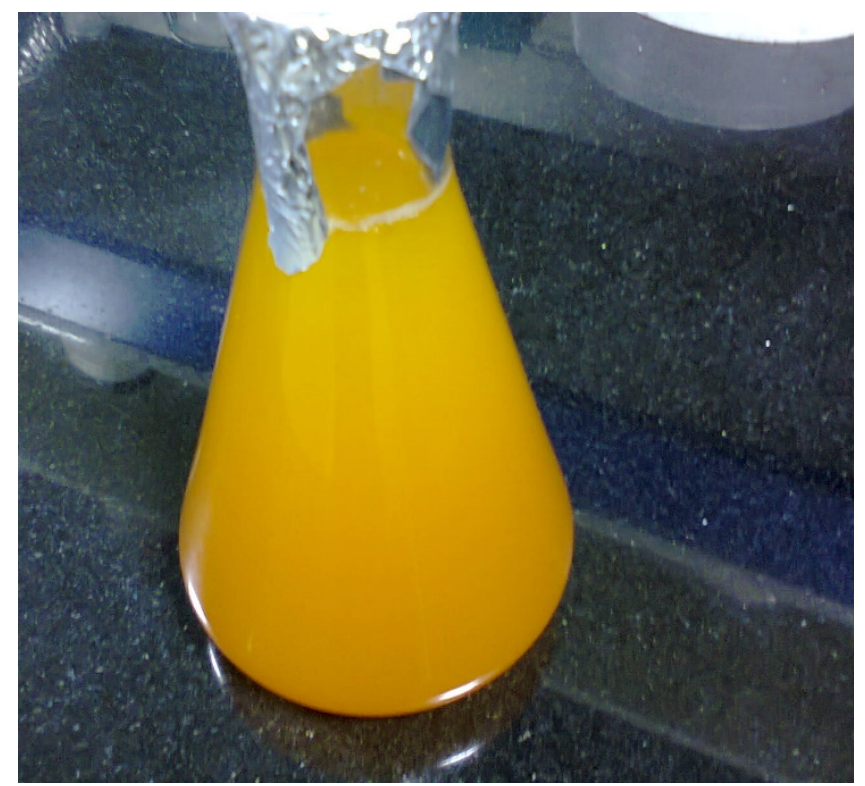

S1: GO dispersed in deionized water. 

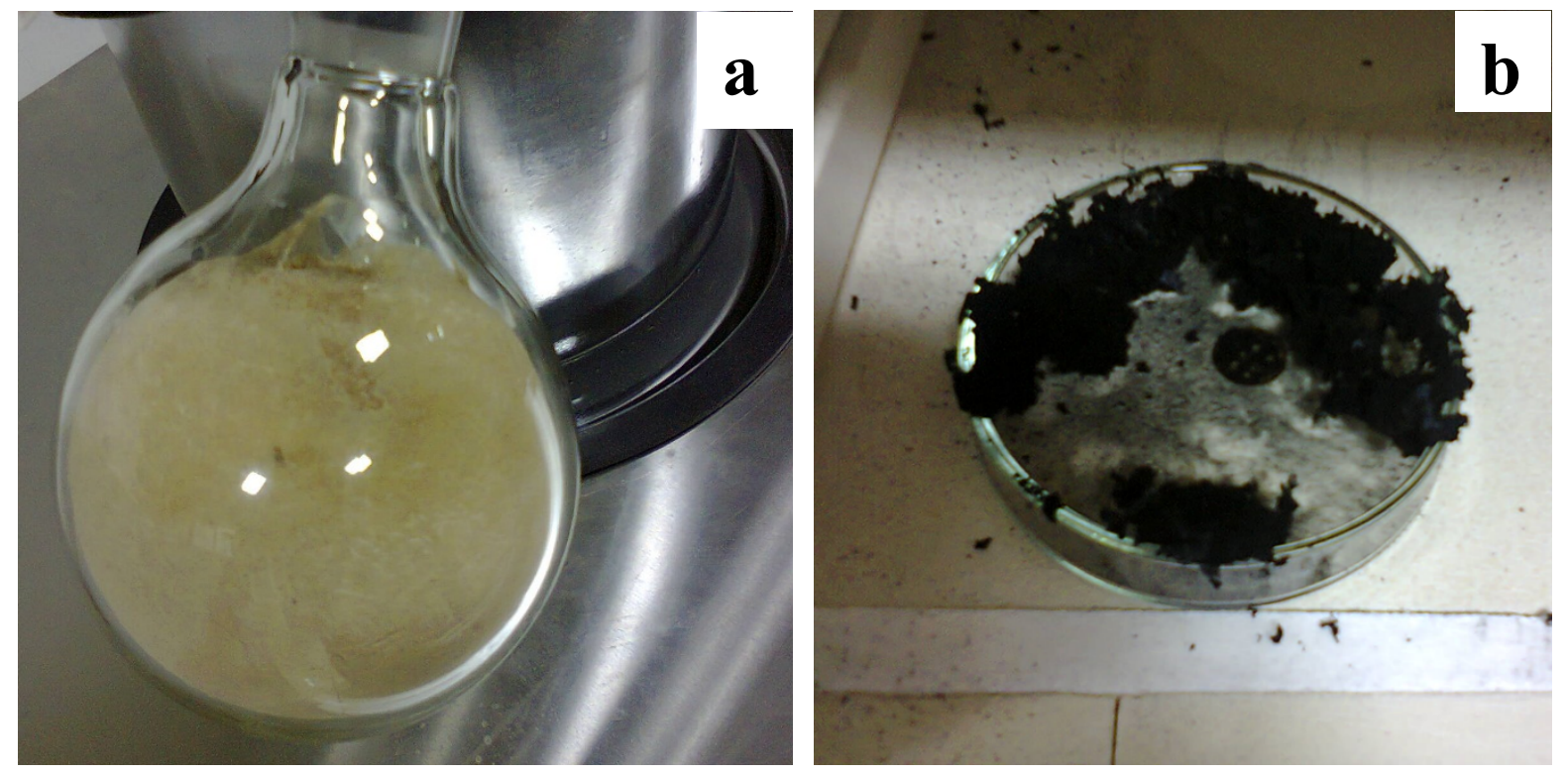

S2: GO after lyophilization (a) and annealed GO (b).

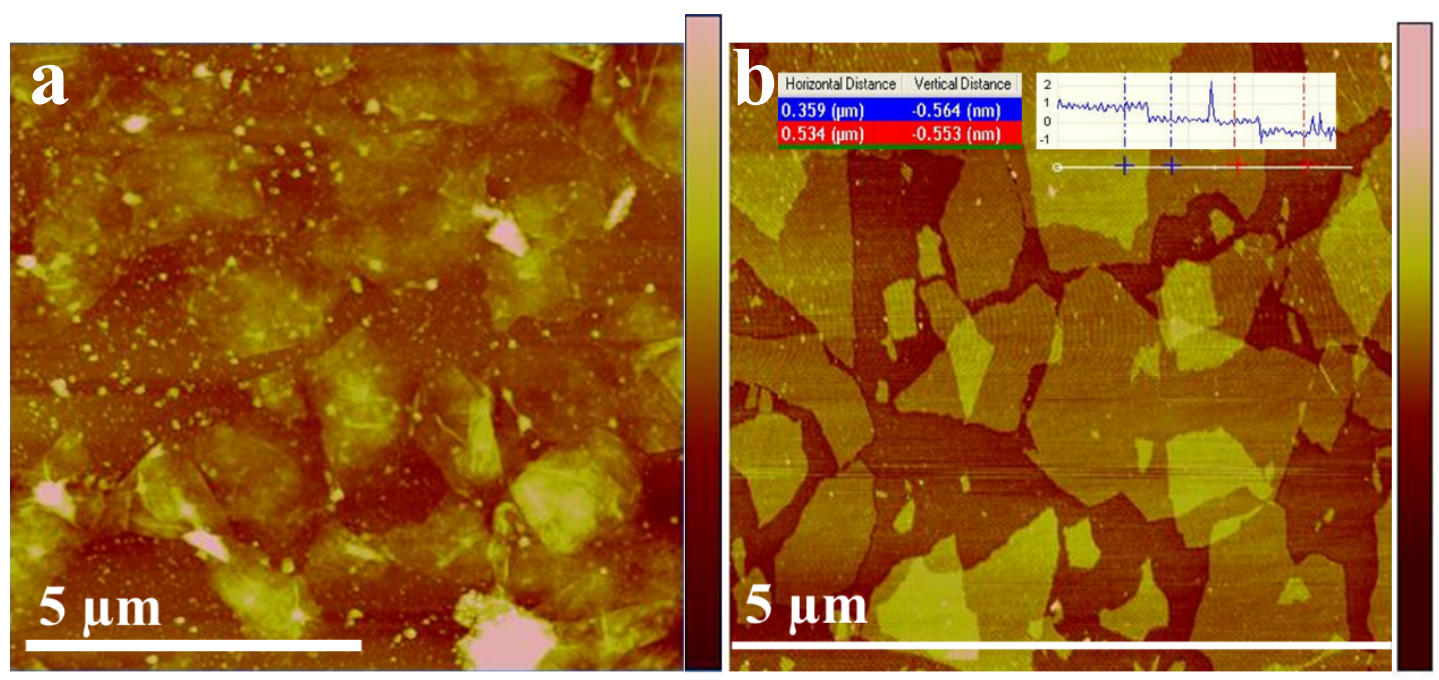

S3: AFM images of GO (a) and GO-400 (b). Bar height for figure (a) and (b) is $100 \mathrm{~nm}$ and $10 \mathrm{~nm}$ respectively.

For AFM measurements, much diluted solutions of GO and GO-400 in water were prepared by ultra-sonication. In figure S3 (a), GO sheets are seen to be having wrinkles. The reason behind this wrinkling is the presence of oxygen functionalities which alter the planer structure of 
graphene. Whereas figure S3 (b) clearly shows the smooth structure owing to the, reduction process after the removal of the functional groups and restoration of the planner structure. The non-uniformity in the lateral dimensions of sheets may be due to the breaking of sheets due to exfoliation and ultra-sonication process.

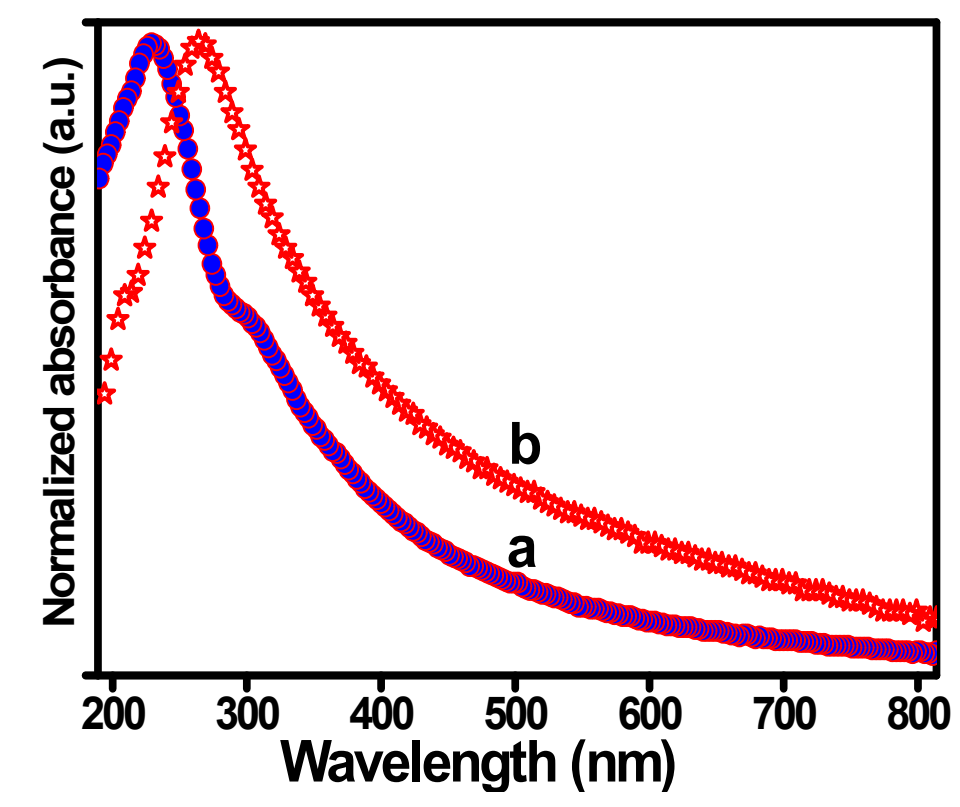

S4: UV-Vis spectra of GO (a) and reduced GO (b).

\section{UV-Vis spectroscopy results:}

UV-Vis absorption measurements were carried out for both as prepared GO and GO-400. In figure S4 (curve a), the two peaks representing the formation of GO were observed at around 230 $\mathrm{nm}$ and a shoulder around $300 \mathrm{~nm}$. The former peak is related to $\pi-\pi^{*}$ transition of $\mathrm{C}=\mathrm{C}$ bonds and later is ascribed as $n-\pi^{*}$ transitions of $\mathrm{C}=\mathrm{O}$ bonds [1]. The reason for shoulder for $\mathrm{C}=\mathrm{O}$ bonds is the higher molar absorptivity of $\pi-\pi^{*}$ transition as compared to $n-\pi^{*}$ transition. The peak related to $\pi-\pi^{*}$ transitions was significantly red shifted after the reduction process and $n-\pi^{*}$ 
transitions related absorption was not observed [curve b]. The red shift can be justified on the basis of the fact that the size of $\mathrm{C}=\mathrm{C}$ network has been increased.

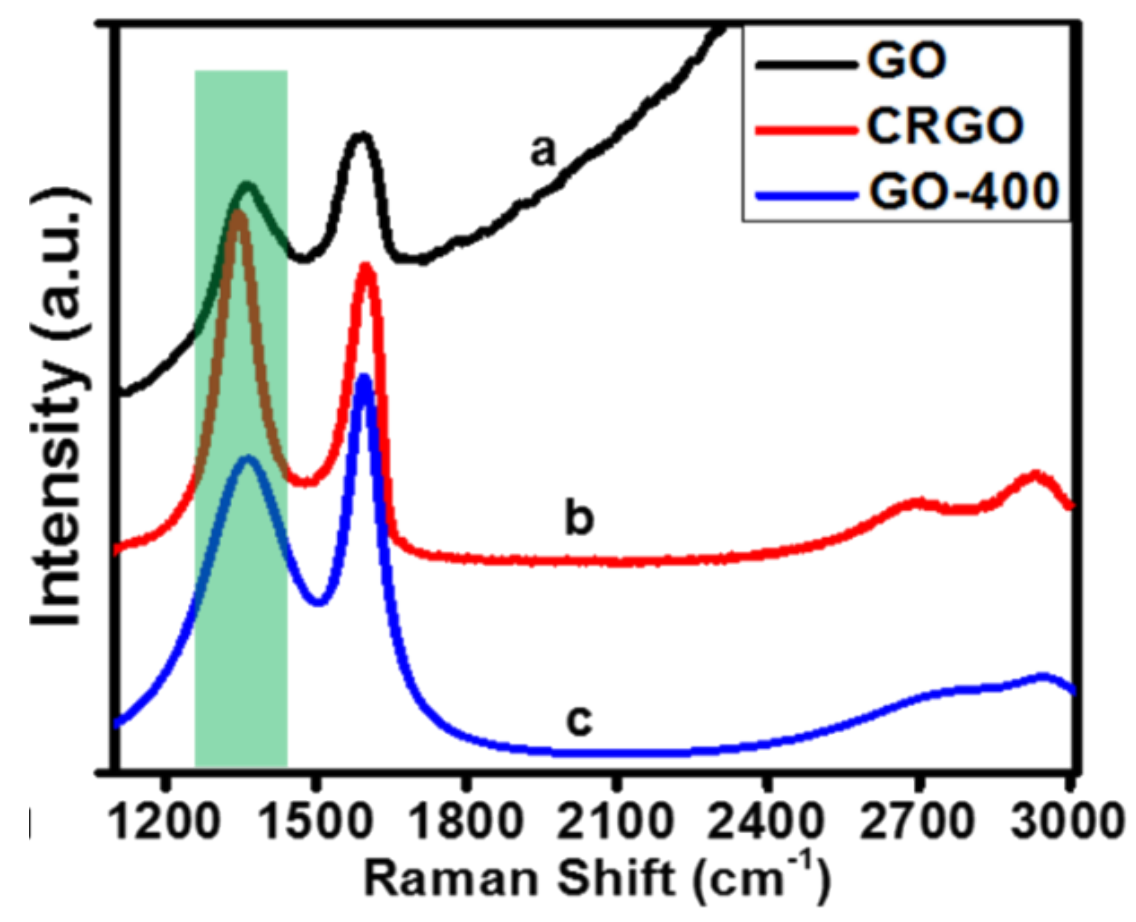

S5: Comparison of Raman spectra of GO (curve a), chemically reduced GO (CRGO) (curve b) and GO-400 (curve c).

S5 represents the Raman spectra of GO, CRGO and GO-400 samples. The defects in the final product were minimized in GO-400 sample (green highlighted region). The decreased $\mathrm{I}_{\mathrm{D}} / \mathrm{I}_{\mathrm{G}}$ ratio reflects the quality of graphene and this ratio was found to be less for GO-400 sample as compared to CRGO sample indicating that the sp2 domain size is much greater in GO-400 which in turn indicated to a better quality of graphene.

\section{Reference:}

1. G. Eda, and M. Chhowalla, Adv. Mat., 2010, 22, 2392-415. 
